\title{
Lateral positioning of InGaAs quantum dots using a buried stressor
}

Cite as: Appl. Phys. Lett. 100, 093111 (2012); https://doi.org/10.1063/1.3691251

Submitted: 30 September 2011 • Accepted: 14 February 2012 • Published Online: 01 March 2012

\section{A. Strittmatter, A. Schliwa, J.-H. Schulze, et al.}

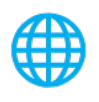

\section{ARTICLES YOU MAY BE INTERESTED IN}

Development of site-controlled quantum dot arrays acting as scalable sources of indistinguishable photons

APL Photonics 5, 096107 (2020); https://doi.org/10.1063/5.0013718

Lithographic alignment to site-controlled quantum dots for device integration Applied Physics Letters 92, 183101 (2008); https://doi.org/10.1063/1.2920189

Resonance fluorescence of a site-controlled quantum dot realized by the buried-stressor growth technique

Applied Physics Letters 110, 111101 (2017); https://doi.org/10.1063/1.4978428

耳QBLOX

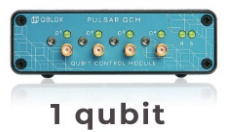

Shorten Setup Time Auto-Calibration More Qubits

Fully-integrated Quantum Control Stacks Ultrastable DC to $18.5 \mathrm{GHz}$ Synchronized $<<1$ ns Ultralow noise

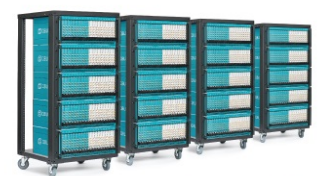

100s qubits

visit our website > 


\title{
Lateral positioning of InGaAs quantum dots using a buried stressor
}

\author{
A. Strittmatter, ${ }^{1, \text { a) }}$ A. Schliwa, ${ }^{1}$ J.-H. Schulze, ${ }^{1}$ T. D. Germann, ${ }^{1}$ A. Dreismann, ${ }^{1}$ \\ O. Hitzemann, ${ }^{1}$ E. Stock, ${ }^{1}$ I. A. Ostapenko, ${ }^{1}$ S. Rodt, ${ }^{1}$ W. Unrau, ${ }^{1}$ U. W. Pohl, ${ }^{1}$ \\ A. Hoffmann, ${ }^{1}$ D. Bimberg, ${ }^{1}$ and V. Haisler ${ }^{2}$ \\ ${ }^{1}$ Technische Universität Berlin, Institut für Festkörperphysik, Sekr. EW 5-2, Hardenbergstrasse 36, D-10623 \\ Berlin, Germany \\ ${ }^{2}$ Russian Academy of Sciences, Institute of Semiconductor Physics, pr. Lavrenteva 13, RU-630090 Novosibirsk, \\ Federation of Russia
}

(Received 30 September 2011; accepted 14 February 2012; published online 1 March 2012)

\begin{abstract}
We present a "bottom-up" approach for the lateral alignment of semiconductor quantum dots (QDs) based on strain-driven self-organization. A buried stressor formed by partial oxidation of $(\mathrm{Al}, \mathrm{Ga}) \mathrm{As}$ layers is employed in order to create a locally varying strain field at a $\mathrm{GaAs}(001)$ growth surface. During subsequent strained layer growth, local self-organization of (In,Ga)As QDs is controlled by the contour shape of the stressor. Large vertical separation of the QD growth plane from the buried stressor interface of $150 \mathrm{~nm}$ is achieved enabling high optical quality of QDs. Optical characterization confirms narrow QD emission lines without spectral diffusion. (C) 2012 American Institute of Physics. [http://dx.doi.org/10.1063/1.3691251]
\end{abstract}

The deterministic alignment of quantum dots (QDs) during an epitaxial growth process is mandatory for electronic and optoelectronic devices ${ }^{1}$ based on single QDs, for example, single photon detectors ${ }^{2}$ and non-classical light emitters. ${ }^{3}$ The self-organized formation of coherently strained islands, e.g., QDs, by the growth of strained layers in the "Stranski-Krastanow" growth regime is a consequence of the total energy minimization of the strained layer system. ${ }^{4-6}$ QDs are formed if the strain energy relieved by island formation surpasses the energy cost associated with newly formed surfaces and edges. ${ }^{7}$ Therefore, a selective formation of QDs on a surface will occur if the surface exhibits sites of increased strain energy, higher strain energy relief, or lower facet formation energy during growth of a strained layer. Current techniques for QD positioning generally deploy nanometer-scale lithography techniques like electron beam lithography, ${ }^{8}$ focused ion beam lithography, ${ }^{9}$ local oxidation, ${ }^{10}$ or nano-imprinting ${ }^{11}$ in order to define nanometersized areas as exclusive nucleation sites prior to the growth of quantum dots. All these "top-down" approaches share a number of difficulties, which impact the structural and optical properties of the quantum dots. First, deterministic quantum dot nucleation is possible only within very close vertical proximity to the structural patterning. An often reported problem is the missing of QDs at shallow holes patterned on a growth surface. ${ }^{8,9}$ Since the patterning involves etching of the surface or other invasive means, the quantum dots will be surrounded by defect sites which degrade their structural and optical quality. ${ }^{12}$ Even though sophisticated cleaning procedures are applied prior to growth, the QDs show inferior optical qualities in terms of spectral linewidth and radiative efficiency as compared to QDs grown on planar substrate surfaces. ${ }^{8,9}$ Second, these technologies are not easily scalable to large wafer sizes because of the involved delicate nanometer-scale processing steps. Third, there is no self-alignment of an electrical current path with respect to

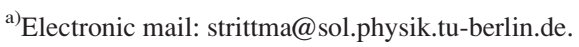

the QDs in order to efficiently inject charge carriers into single quantum dots.

We report on a "bottom-up" approach employing a local modification of the free energy of a GaAs(001) surface using spatially modulated strain fields. These strain fields emanate from the interfaces of a buried $\mathrm{Al}_{\mathrm{x}} \mathrm{Ga}_{1-\mathrm{x}} \mathrm{As} / \mathrm{AlO}_{\mathrm{x}}$ stressor obtained by partial oxidation of AlGaAs. ${ }^{13,14}$ The strain field is created by altering the volume of the initial AlGaAs upon oxidation. During subsequent InGaAs strained layer growth the InAs growth is preferred at the local tensile strain maxima of the GaAs surface. Consequently, local QD formation is observed. For properly reduced size of the stressor, controlled growth of single QDs at the center of the mesa is obtained. We first analyze the stressor-induced strain distribution at a $\mathrm{GaAs}(001)$ surface and report afterwards on the positioning of InGaAs QD using buried stressors.

The strain distribution as obtained by a continuummechanical model at the surface of a circular mesa structure for a rectangular $\mathrm{Al}_{\mathrm{x}} \mathrm{Ga}_{1-\mathrm{x}} \mathrm{As} / \mathrm{AlO}_{\mathrm{x}}$ stressor buried $100 \mathrm{~nm}$ beneath a $\mathrm{GaAs}(001)$ surface is shown in Fig. 1. The upper panel shows a schematic of the structure used for modeling. An isotropic contraction of the unit cell volume by $7 \%$ upon oxidation is assumed for all calculations. As a result of the volume contraction, the region above the oxidized buried layer is compressively strained. However, the volume contraction exerts a tensile force onto the region at the boundaries between the oxide and the AlAs. The result as shown in the lower panel of Fig. 1 is an inhomogeneous strain distribution $\varepsilon(\mathrm{x}, \mathrm{y})$ at the $\operatorname{GaAs}(001)$ surface. On the vertical axis the strain distribution $\varepsilon=\varepsilon_{\mathrm{xx}}+\varepsilon_{\mathrm{yy}}$ at the surface is displayed. Two cross-sectional line scans of the strain distribution of rectangular apertures with 2 and $1 \mu \mathrm{m}$ side length reveal the impact of the aperture size on the strain distribution. Whereas two separate maxima of the tensile in-plane strain are located above the aperture region with $2 \mu \mathrm{m}$ side length only a single tensile strain maximum is found for the aperture $1 \mu \mathrm{m}$ side length. For the given structure with a $100 \mathrm{~nm}$ GaAs layer thickness on top of the oxide, the magnitude of 


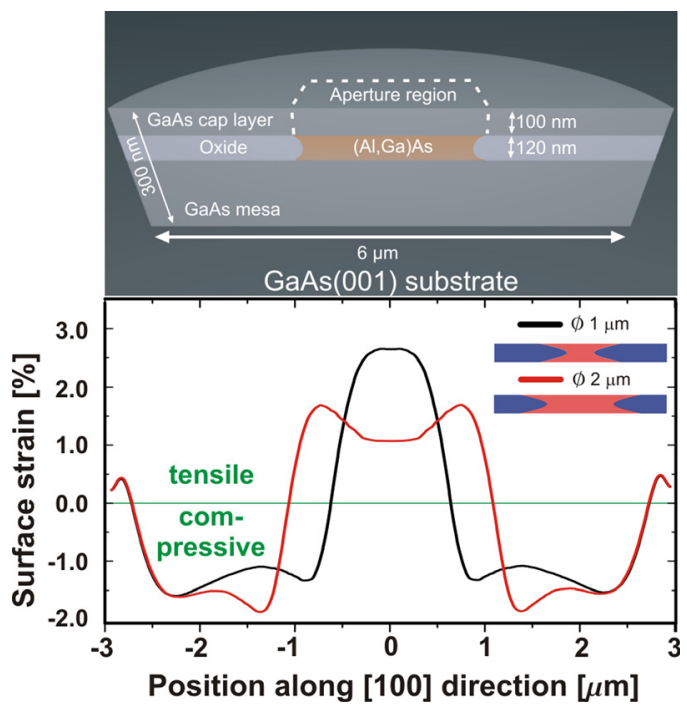

FIG. 1. (Color online) (Upper panel) Schematic of the buried stressor structure as used for strain calculation. (Lower panel) Calculated sum of the in-plane strain components $\left(\varepsilon=\varepsilon_{\mathrm{xx}}+\varepsilon_{\mathrm{yy}}\right)$ at the surface of a $\mathrm{GaAs}(001)$ substrate for a buried $\mathrm{AlAs} / \mathrm{AlO}_{\mathrm{x}}$ stressor structure covered by $100 \mathrm{~nm}$ GaAs. For square-like aperture of $2 \mu \mathrm{m}$ side length (black line) the maximum inplane strain is surrounding the inner part of the aperture. For an aperture side length of $1 \mu \mathrm{m}$ (red line) maximum tensile strain is found at the center of the aperture.

the interfacial strain $\left(\varepsilon_{\mathrm{xx}}\right.$ or $\left.\varepsilon_{\mathrm{yy}}\right)$ is of the order of $1 \%-1.5 \%$. As the QD formation in the $\operatorname{In}_{\mathrm{x}} \mathrm{Ga}_{1-\mathrm{x}} \mathrm{As} / \mathrm{GaAs}$ system is driven by interfacial lattice mismatch $(\varepsilon \sim 3 \%-7 \%$ for $\mathrm{x}>0.30)$ this magnitude of the surface strain is significant for the QD growth. Since InAs has a larger lattice constant than GaAs the points of maximum tensile strain at the GaAs surface correspond to points of lowest strain for the InAs layer. The inhomogeneous strain distribution will therefore affect growth of both GaAs and InAs layers since atoms will move to the minima of their respective surface strain energy during growth. As the total amount of InAs triggers the onset of quantum dot formation, ${ }^{15}$ selective growth of QDs at the aperture boundaries is expected. From the simulation data, the width of the tensile strain maximum is about $300 \mathrm{~nm}$ which sets the spatial resolution for QD alignment to a circle of about $300 \mathrm{~nm}$ diameter. Since the oxide layer defines a current path through the aperture in vertical pn-junction devices, the QDs are inherently self-aligned to this current path.

The process for the stressor fabrication starts with the growth of the epitaxial stressor structure consisting of $40 \mathrm{~nm}$ $\mathrm{Al}_{0.90} \mathrm{Ga}_{0.10} \mathrm{As} / 40 \mathrm{~nm} \mathrm{AlAs} / 40 \mathrm{~nm} \mathrm{Al}_{0.90} \mathrm{Ga}_{0.10} \mathrm{As}$ which is covered by $50 \mathrm{~nm}$ GaAs. After growth, circular mesas are formed by dry etching and finally, the AlAs layer is oxidized in a water vapor/nitrogen ambient from the mesa edges towards the center (see Fig. 1). The fabricated circular mesas are $10-25 \mu \mathrm{m}$ in diameter. Upon an oxidation time of approximately $10 \mathrm{~min}$, the oxidation depth is about $7 \mu \mathrm{m}$ leading to apertures in the oxide layer for $d_{\text {mesa }}>14 \mu \mathrm{m}$, which are centered with respect to the mesa boundaries. The aperture sizes scale with the mesa diameter from $<1 \mu \mathrm{m}$ for $\mathrm{d}_{\text {mesa }}<16 \mu \mathrm{m}$ to $\sim 2 \mu \mathrm{m}$ for $\mathrm{d}_{\text {mesa }}=20 \mu \mathrm{m}$. Prior to QD formation sample surfaces are annealed at $720^{\circ} \mathrm{C}$ and a $50 \mathrm{~nm}$ GaAs buffer layer is grown at $690^{\circ} \mathrm{C}$ which is standard for GaAs growth on un-patterned substrates. For QD formation,
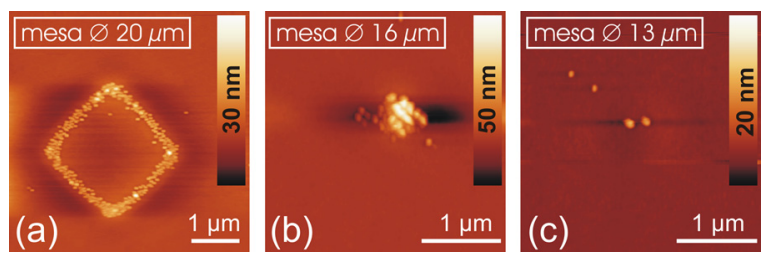

FIG. 2. (Color online) Atomic-force microscopy images of the center regions of mesa structures with (a) $20 \mu \mathrm{m}$, (b) $14 \mu \mathrm{m}$, and (c) $13 \mu \mathrm{m}$ diameter overgrown with quantum dots. QDs appear as hillocks and show clear lateral ordering in a square-like pattern (a) according to the shape of the $2 \times 2 \mu \mathrm{m}^{2}$ rectangular stressor. For aperture sizes less than $1 \mu \mathrm{m}$, a cluster of QDs is found (b). The number of aligned QDs at the mesa center is reduced to 2 quantum dots for aperture sizes of less than $500 \mathrm{~nm}$ (c). Scale bars in the figures indicate the height scale.

approximately 2 monolayers of $\mathrm{In}_{0.5} \mathrm{Ga}_{0.5} \mathrm{As}$ are deposited onto the GaAs surface by metalorganic vapor phase epitaxy. Thereby, the vertical separation of the QD growth plane from the stressor is about $150 \mathrm{~nm}$ which is much larger than for QDs grown on patterned nano-holes, for example.

Figures 2(a)-2(c) are atomic force microscopy (AFM) surface images taken at the center of mesas with diameters of 20,14 , and $13 \mu \mathrm{m}$ exhibiting apertures of $2 \mu \mathrm{m},<1 \mu \mathrm{m}$, and $<500 \mathrm{~nm}$ side length, respectively. For this experiment, the InGaAs QDs are buried beneath the surface by only $4 \mathrm{~nm}$ GaAs to distinguish the QDs by hillocks on the surface. For the $2 \times 2 \mu \mathrm{m}^{2}$ aperture (Fig. 2(a)), a square-like alignment of the QDs is observed while a QD cluster appears for the $14 \mu \mathrm{m}$ mesa (Fig. 2(b)). With an aperture size of less than $500 \mathrm{~nm}(13 \mu \mathrm{m}$ mesa diameter) only two QDs are left at the aperture center (Fig. 2(c)) in agreement with our theoretical modeling. Optical properties of the QDs are investigated by cathodoluminescence and micro-photoluminescence spectroscopy. The QD luminescence distribution is very well correlated to the alignment found in the AFM images. For example, $20 \mu \mathrm{m}$ large mesas show QD luminescence distribution along the boundaries of a $2 \times 2 \mu \mathrm{m}^{2}$ square (not shown). Figure 3 shows the spatial distribution of QD emission $(1.28-1.32 \mathrm{eV})$ on a $15 \mu \mathrm{m}$ mesa and a local spectrum taken at the center to demonstrate the high optical quality of the QDs. From the AFM analysis (Fig. 2(b)), a cluster of QDs is expected to be formed at the center of the mesa. Amongst other QD emission lines, a spectrally separated single emission line is selected for demonstration purposes. This line exhibits a full-width at half-maximum of $160 \mu \mathrm{eV}$

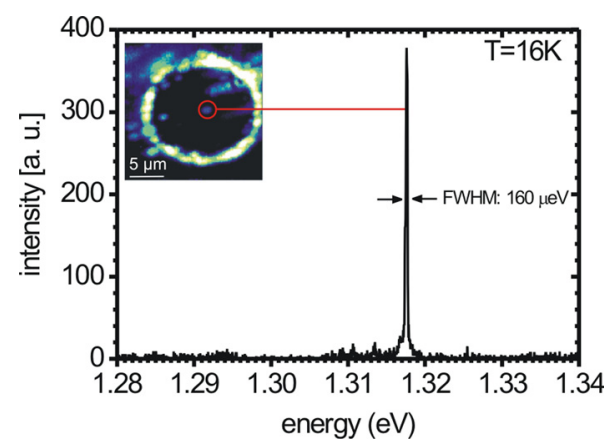

FIG. 3. (Color online) Individual QD emission line in the center of a $15 \mu \mathrm{m}$ mesa with $160 \mu \mathrm{eV} \mathrm{FWHM} \mathrm{as} \mathrm{indicated} \mathrm{in} \mathrm{the} \mathrm{inset.} \mathrm{Inset:} \mathrm{spatial} \mathrm{distribu-}$ tion of QD luminescence between 1.28 and $1.32 \mathrm{eV}$. The local value of maximum intensity is encoded in false colors. Additional QDs have formed in the region of reduced strain at the mesa's edge. 
which is limited by the spectral resolution of the setup. The value compares well with some of the best reported values to date. ${ }^{8,16}$ In Ref. 8, an average QD emission linewidth of $132 \mu \mathrm{eV}$ is reported, and in Ref. 14, the linewidth is about $100 \mu \mathrm{eV}$. The intensity of the spectrum is comparable to that of QD samples grown on planar substrates, demonstrating high optical quality of the aligned QDs. Very little spectral diffusion of QD emission lines is observed on individual emission lines which indicates high quality of the surrounding GaAs material.

A "bottom-up" technique for QD positioning based on lateral self-organization above a buried stressor is developed. Deterministic alignment of InGaAs QDs at large vertical distance from a buried $\mathrm{Al}_{\mathrm{x}} \mathrm{Ga}_{1-\mathrm{x}} \mathrm{As} / \mathrm{AlO}_{\mathrm{x}}$ stressor is achieved. QDs are located above the inner regions of the buried oxide aperture which inherently defines an electrical current path through the QDs in pn-junction devices. Optical characterization yields narrow QD emission lines with high luminescence intensity and little spectral diffusion.

We gratefully acknowledge helpful discussions with D. Gershoni, Technion, Haifa (Israel). Financial support for this work is received by the Deutsche Forschungsgemeinschaft within the Collaborative Research Center 787 "Nanophotonic devices" (projects A2, A5, and C1).
${ }^{1}$ J. V. Barth, G. Costantini, and K. Kern, Nature 437(7059), 671 (2005).

${ }^{2}$ S. Komiyama, O. Astafiev, V. Antonov, T. Kutsuwa, and H. Hirai, Nature 403(6768), 405 (2000).

${ }^{3}$ A. J. Shields, Nat. Photonics 1(4), 215 (2007).

${ }^{4}$ E. Pehlke, N. Moll, A. Kley, and M. Scheffler, Appl. Phys. A 65(6), 525 (1997).

${ }^{5}$ V. A. Shchukin and D. Bimberg, Appl. Phys. A 67(6), 687 (1998).

${ }^{6}$ N. Moll, M. Scheffler, and E. Pehlke, Phys. Rev. B 58(8), 4566 (1998).

${ }^{7}$ V. A. Shchukin, D. Bimberg, T. P. Munt, and D. E. Jesson, Phys. Rev. B 70(8), 085416 (2004).

${ }^{8}$ J. Skiba-Szymanska, A. Jamil, I. Farrer, M. B. Ward, C. A. Nicoll, D. J. P. Ellis, J. P. Griffiths, D. Anderson, G. A. C. Jones, D. A. Ritchie, and A. J. Shields, Nanotechnology 22(6), 065302 (2011).

${ }^{9}$ J. Lee, T. W. Saucer, A. J. Martin, D. Tien, J. M. Millunchick, and V. Sih, Nano Lett. 11(3), 1040 (2011).

${ }^{10}$ J. Martin-Sanchez, Y. Gonzalez, L. Gonzalez, M. Tello, R. Garcia, D. Granados, J. M. Garcia, and F. Briones, J. Cryst. Growth 284(3-4), 313 (2005).

${ }^{11}$ T. V. Hakkarainen, J. Tommila, A. Schramm, A. Tukiainen, R. Ahorinta, M. Dumitrescu, and M. Guina, Appl. Phys. Lett. 97(17), 173107 (2010).

${ }^{12}$ M. Bayer and A. Forchel, Phys. Rev. B 65(4), 041308 (2002).

${ }^{13}$ K. D. Choquette, K. M. Geib, C. I. H. Ashby, R. D. Twesten, O. Blum, H. Q. Hou, D. M. Follstaedt, B. E. Hammons, D. Mathes, and R. Hull, IEEE J. Sel. Top. Quantum 3(3), 916 (1997).

${ }^{14}$ A. Lochmann, E. Stock, O. Schulz, R. Hopfer, D. Bimberg, V. A. Haisler, A. I. Toropov, A. K. Bakarov, and A. K. Kalagin, Electron. Lett. 42(13), 774 (2006).

${ }^{15}$ H. Toyoshima, T. Niwa, J. Yamazaki, and A. Okamoto, Appl. Phys. Lett. 63(6), 821 (1993).

${ }^{16}$ A. Mohan, P. Gallo, M. Felici, B. Dwir, A. Rudra, J. Faist, and E. Kapon, Small 6(12), 1268 (2010). 